\title{
An increase in the proportion of lymphocytes is necessary for tumor reduction in patients with non-small-cell lung cancer treated with immune checkpoint inhibitors
}

\author{
Satoshi Hirano ${ }^{1 *}$, Hiroyuki Amano ${ }^{2}$, Tetsuo Fujita ${ }^{2}$, Sukeyuki Nakamura ${ }^{2}$, Nami Hayama², Yuri Suzuki ${ }^{2}$, Makoto Nakamura ${ }^{2}$ and Hiroshi \\ Tabeta $^{3}$ \\ ${ }^{1}$ Department of Medical Oncology, Funabashi Municipal Medical Center, Japan \\ ${ }^{2}$ Department of Respiratory Medicine, Funabashi Municipal Medical Center, Japan \\ ${ }^{3}$ Department of Palliative Care Medicine, Funabashi Municipal Medical Center, Japan
}

\begin{abstract}
Background: Immunotherapy with immune checkpoint inhibitor is one of the standard therapies for advanced non-small-cell lung cancer (NSCLC). However, the proportion of patients who benefit from this treatment is limited. We evaluated the utility of changes in the percentage of peripheral lymphocytes as a predictor of tumor response.

Patients and methods: We conducted a retrospective study of a single center. We included consecutive patients with advanced NSCLC treated with nivolumab or pembrolizumab from January 2016 to March 2018 at Funabashi Municipal Medical Center. Changes in the percentage of lymphocytes in peripheral blood 6 weeks after treatment with immune checkpoints inhibitor from baseline values were compared between patients with tumor shrinkage and all other patients.

Results: Fifty-three patients were evaluable. The percentage of lymphocytes in tumor-shrinking patients increased from $18 \%$ at baseline to $27 \%$ (p<0.001). On the other hand, percentages in patients without tumor shrinkage were $21 \%$ and $20 \%$, respectively $(\mathrm{p}=0.472)$.

Conclusions: An increase in the percentage of lymphocytes in peripheral blood at 6 weeks might offer a biomarker for predicting the clinical benefit of NSCLC patients undergoing immune checkpoint inhibitor therapy.
\end{abstract}

\section{Introduction}

Immunotherapy with immune checkpoint inhibitors such as nivolumab and pembrolizumab is considered to be one of the standard treatments for advanced non-small-cell lung cancer (NSCLC) [1-3]. However, the proportion of patients who benefit from this treatment is limited. For those who fail this treatment, another anticancer therapy is needed as soon as possible to avoid further systemic deterioration by the tumor. In this study, the increase in the percentage of peripheral lymphocytes from baseline to 6 weeks after immune checkpoint inhibitor treatment was evaluated to clarify the utility of this marker in predicting tumor reduction.

\section{Material and methods}

The medical records of patients with advanced NSCLC treated with nivolumab or pembrolizumab at Funabashi Municipal Medical Center between January 2016 and March 2018 were retrospectively reviewed. Nivolumab was administered at a dose of $3 \mathrm{mg} / \mathrm{kg}$ once every 2 weeks, and pembrolizumab was administered at a dose of $200 \mathrm{mg} /$ body once every 3 weeks. Collected data included the following: age, sex, smoking history, Eastern Cooperative Oncology Group Performance Status (ECOG PS) at the time of initiating treatment, histology, clinical stage based on the seventh edition of the TNM classification of Malignant Tumors by the International Union Against Cancer and the American Joint Committee on Cancer, white blood cell count (WBC), percentages of neutrophils and lymphocytes just before administration and after about 6 weeks of the administration of immune checkpoint inhibitors. Response to treatment was assessed by the treating physician. Patients were included in the tumor shrinkage group if contrast-enhanced computer tomography showed a decrease in tumor volume after administration of the immune checkpoint inhibitor. Changes in the percentage of lymphocytes in peripheral blood 6 weeks after treatment with the immune checkpoint inhibitor from baseline value were compared between patients with tumor shrinkage and other patients. Characteristics of patients were compared using the chi-square test or Fisher's exact test. For comparisons of the WBC and percentages of neutrophils and lymphocytes before and 6 weeks after treatment, the paired $t$ test was performed. The Mann-Whitney test was used for comparisons between the tumor shrinkage and non-shrinkage groups. Values of $\mathrm{P}<0.05$ were considered statistically significant in all analyses. All statistical analyses were carried out using $\mathrm{PASW}^{*}$ Statistics version 18 .

${ }^{*}$ Correspondence to: Satoshi Hirano, MD, $\mathrm{PhD}, 1-21-1$ Kanasugi, Funabashi, Chiba, Japan, Tel: 81474383321; Fax: 81474302518; E-mail: shirano@mmc. funabashi.chiba.jp

Key words: NSCLC, immune checkpoint inhibitor, lymphocyte proportion, antitumor effect, predictive marker

Received: September 05, 2018; Accepted: September 24, 2018; Published: September 27, 2018 
Hirano S (2018) An increase in the proportion of lymphocytes is necessary for tumor reduction in patients with non-small-cell lung cancer treated with immune checkpoint inhibitors

\section{Results}

Fourteen patients were excluded due to poor data collection or early disease progression. Eventually, 60 patients were included. Tumor shrinkage was obtained in 24 patients. Antitumor effects could not be evaluated in 7 patients. Baseline characteristics of patients are shown in Table 1.

Mean baseline WBC, percentage of neutrophils and percentage of lymphocytes were $6800 / \mu \mathrm{L}, 70 \%$ and $19 \%$, respectively. At 6 weeks after the start of treatment, these values were $7200 / \mu \mathrm{L}, 64 \%$ and $24 \%$, respectively. An increase in the percentage of lymphocytes and a decrease in the percentage of neutrophils were demonstrated (data not shown).

In the tumor shrinkage group, an increase in the percentage of lymphocytes after 6 weeks of treatment was demonstrated in all patients, with a mean value of $27 \%$ compared to the baseline of $18 \%(\mathrm{p}<0.001)$ (Figure 1). On the other hand, in patients without any tumor shrinkage, percentages at baseline and 6 weeks were $21 \%$ and $20 \%$, respectively $(\mathrm{p}=0.472)$. A decrease in the percentage of neutrophils from $71 \%$ at baseline to $60 \%$ at 6 weeks $(\mathrm{p}=0.006)$ was seen in patients with tumor shrinkage and an increase of WBC from $6700 / \mu \mathrm{L}$ to $7700 / \mu \mathrm{L}$ in patients without tumor shrinkage $(\mathrm{p}<0.001)$ were also demonstrated.

\section{Discussion}

PD-L1 immunohistochemistry is considered as one of the most reliable predictors for immune therapy. However, not all patients showing positive results from PD-L1 staining do not necessarily respond to immune therapy. For such patients, another antitumor treatment with cytotoxic anticancer drugs is needed as soon as possible. Our study showed a marked increase in the percentage of lymphocytes after 6 weeks in patients with sensitivity to immune checkpoint inhibitor therapy. In other words, patients with no increase in the percentage of

Table 1. Patient characteristics

\begin{tabular}{|c|c|c|c|}
\hline Characteristics & $\begin{array}{c}\text { Patients without } \\
\text { tumor regression } \\
(n=24)\end{array}$ & $\begin{array}{c}\text { Patients without } \\
\text { tumor regression } \\
(n=29)\end{array}$ & P value \\
\hline \multicolumn{4}{|l|}{ Age, years } \\
\hline Median (Range) & $70.5(59-82)$ & $70(47-84)$ & \\
\hline \multicolumn{3}{|l|}{ Sex, n (\%) } & 0.751 \\
\hline Male & $18(75 \%)$ & $23(79.3 \%)$ & \\
\hline Female & $6(25 \%)$ & $6(20.7 \%)$ & \\
\hline \multicolumn{3}{|c|}{ ECOG performance status score, $\mathrm{n}(\%)$} & 0.775 \\
\hline 0 & $11(45.8 \%)$ & $11(37.9 \%)$ & \\
\hline 1 & $12(50 \%)$ & $16(55.2 \%)$ & \\
\hline 2 & $1(4.2 \%)$ & $2(6.9 \%)$ & \\
\hline \multicolumn{3}{|l|}{ Smoking status, $\mathrm{n}(\%)$} & 0.715 \\
\hline Current or former smoker & $21(87.5 \%)$ & $24(82.8 \%)$ & \\
\hline Never smoked & $3(12.5 \%)$ & $5(17.2 \%)$ & \\
\hline \multicolumn{3}{|l|}{ Tumor histologic type, $\mathrm{n}(\%)$} & 0.634 \\
\hline Squamous & $8(33.3 \%)$ & $12(41.4 \%)$ & \\
\hline Non-squamous & $16(67.7 \%)$ & $17(58.6 \%)$ & \\
\hline \multicolumn{3}{|c|}{ Number of prior therapies, $\mathrm{n}(\%)$} & 0.138 \\
\hline 0 & $7(29.2 \%)$ & $3(10.3 \%)$ & \\
\hline 1 & $8(33.3 \%)$ & $19(65.5 \%)$ & \\
\hline 2 & $9(37.5 \%)$ & $7(24.2 \%)$ & \\
\hline \multicolumn{3}{|l|}{ Immune checkpoint inhibitor } & 0.358 \\
\hline Nivolumab & $16(66.7 \%)$ & $23(79.3 \%)$ & \\
\hline Pembrolizumab & $8(33.3 \%)$ & $6(20.7 \%)$ & \\
\hline
\end{tabular}

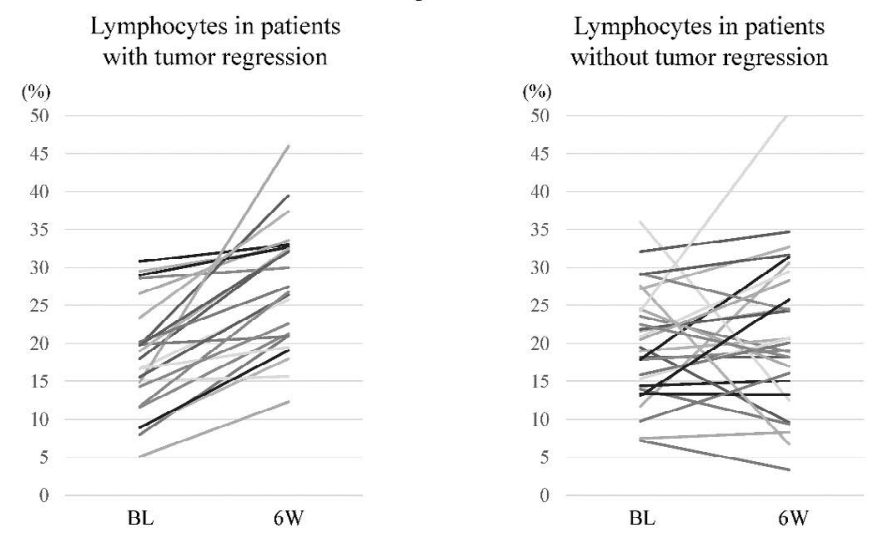

Figure 1. Change in lymphocyte proportion over 6 weeks in each patient. An increase in lymphocyte proportion was demonstrated in all patients with tumor shrinkage (BL: Baseline; 6W: 6 weeks after administrating the immune checkpoint inhibitor)

lymphocytes have a very low possibility of tumor shrinkage. We propose that the increase in the percentage of lymphocytes in peripheral blood might provide a useful biomarker for predicting clinical benefit in NSCLC patients undergoing immune checkpoint inhibitor therapy.

During viral infection, virus-specific CD8 $\mathrm{T}$ cells are activated and clonal proliferation is induced. Up to $70 \%$ of responding CD8 T cells during primary or secondary infection can be virus-specific. Readministration of the same virus triggers a rapid expansion of memory T cells [4].

As with viral infection, the in vivo proliferation rate of CD8 $\mathrm{T}$ cells transferred after proper stimulation is very fast, calculated as $5.3 \mathrm{~h} /$ cell division in mice with B16 melanoma [5]. Patients with metastatic melanoma who responded during anti-PD-1 therapy also been reported to show proliferation of CD8 T cells at the invasive margins. In addition, an increase in the number of CD8 T cells was associated with response to therapy. Therefore, clonal proliferation of $\mathrm{T}$ cells in tumor tissue is also believed to predict the clinical benefit of immune checkpoint therapy [6-8]. However, repeated biopsy especially in lung cancer patients is not always easy and is sometimes very painful for the patients. Taking these facts into consideration, tumor antigen-specific CD8 T-cell clones in peripheral blood could correlate with anti-tumor responses induced by therapy with immune checkpoint inhibitors. In addition, the standard CD8 T-cell subset consists of $13-40 \%$ of total lymphocytes, and clonal expansion of CD8 T cells would affect the total proportion of lymphocytes. Determining the percentage of lymphocytes in the WBC is very easy and inexpensive. We therefore selected this value as a potential alternative for predicting the efficacy of immune checkpoint inhibitors.

Some reports have described the utility of neutrophil-to-lymphocyte ratio (NLR) as a predictor in NSCLC [9]. NLR was also calculated in our tumor-shrinking patients, but the percentage of lymphocytes provided a more accurate marker (data not shown).

Our study has a few limitations that warrant consideration. First, this study was a small, retrospective study performed at a single institution, with selective bias. Patients who died or changed treatment within 6 weeks were not included in this study. The results are therefore not applicable to patients who are unable to continue immune checkpoint inhibitor therapy for at least 6 weeks. Secondly, the proportion of lymphocytes was likely to be affected by preceding treatments that decreased the number of neutrophils, such as chemotherapy using cytotoxic anticancer drugs or radiotherapy. 
Hirano S (2018) An increase in the proportion of lymphocytes is necessary for tumor reduction in patients with non-small-cell lung cancer treated with immune checkpoint inhibitors

In conclusion, the change in the percentage of lymphocytes in peripheral blood after 6 weeks of immune checkpoint inhibitor therapy might offer a biomarker to predict clinical benefit in NSCLC patients.

\section{Funding}

This research did not receive any specific grants from funding agencies.

\section{References}

1. Brahmer J, Reckamp KL, Baas P, Crinò L, Eberhardt WE, et al. (2015) Nivolumab versus docetaxel in advanced squamous-cell non-small-cell lung cancer. $N$ Engl J Med 373: 123-135. [Crosssref]

2. Borghaei H, Paz-Ares L, Horn L, Spigel DR, Steins M, et al. (2015) Nivolumab versus docetaxel in advanced nonsquamous non-small-cell lung cancer. $N$ Engl J Med 373 : 1627-1639. [Crosssref]

3. Reck M, Rodríguez-Abreu D, Robinson AG, Hui R, Csőszi T, et al. (2016) Pembrolizumab versus chemotherapy for PD-L1-positive non-small-cell lung cancer. N Engl J Med 375: 1823-1833. [Crosssref]
4. Murali-Krishna K, Altman JD, Suresh M, Sourdive DJ, Zajac AJ, et al. (1998) Counting antigen-specific CD8 T cells: a reevaluation of bystander activation during viral infection. Immunity 8: 177-187. [Crosssref]

5. Hwang LN, Yu Z, Palmer DC, Restifo NP (2006) The in vivo expansion rate of properly stimulated transferred CD8+ T cells exceeds that of an aggressively growing mouse tumor. Cancer Res 66: 1132-1138. [Crosssref]

6. Tumeh PC, Harview CL, Yearley JH, Shintaku IP, Taylor EJ, et al. (2014) PD-1 blockade induces responses by inhibiting adaptive immune resistance. Nature 515: 568-571. [Crosssref]

7. Riaz N, Havel JJ, Makarov V, Desrichard A, Urba WJ, et al. (2017) Tumor and microenvironment evolution during immunotherapy with nivolumab. Cell 171: 934949. [Crosssref]

8. Subudhi SK, Aparicio A, Gao J, Zurita AJ, Araujo JC, et al. (2016) Clonal expansion of CD8 T cells in the systemic circulation precedes development of ipilimumab-induced toxicities. Proc Natl Acad Sci USA 113: 11919-11924. [Crosssref]

9. Kiriu T, Yamamoto M, Nagano T, Hazama D, Sekiya R, et al. (2018) The time-series behavior of neutrophil-to-lymphocyte ratio is useful as a predictive marker in nonsmall cell lung cancer. PLoS One 13: e0193018. [Crosssref]

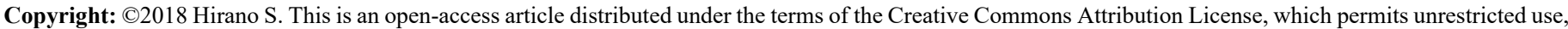
distribution, and reproduction in any medium, provided the original author and source are credited. 